Case Report

\title{
Cervical Spinal Cord Compression: A Rare Presentation of Hepatocellular Carcinoma
}

\author{
Puvanalingam Ayyadurai, ${ }^{1}$ Kanthi Rekha Badipatla, ${ }^{1,2}$ Chukwunonso Chime, ${ }^{1}$ \\ Shiva Arjun,, ${ }^{1,3}$ Pavithra Reddy, ${ }^{1}$ Masooma Niazi, ${ }^{4}$ and Suresh Kumar Nayudu ${ }^{1,2}$ \\ ${ }^{1}$ Department of Medicine, Bronx Lebanon Hospital Center, Affiliated with Icahn School of Medicine at Mount Sinai, Bronx, NY, USA \\ ${ }^{2}$ Division of Gastroenterology, Bronx Lebanon Hospital Center, Affiliated with Icahn School of Medicine at Mount Sinai, \\ Bronx, NY, USA \\ ${ }^{3}$ American University of Caribbean, Cupecoy, Saint Martin \\ ${ }^{4}$ Department of Pathology, Bronx Lebanon Hospital Center, Affiliated with Icahn School of Medicine at Mount Sinai, Bronx, NY, USA
}

Correspondence should be addressed to Puvanalingam Ayyadurai; puvan855@yahoo.co.in

Received 23 November 2016; Accepted 18 January 2017; Published 9 February 2017

Academic Editor: Fumio Imazeki

Copyright (C) 2017 Puvanalingam Ayyadurai et al. This is an open access article distributed under the Creative Commons Attribution License, which permits unrestricted use, distribution, and reproduction in any medium, provided the original work is properly cited.

Hepatocellular carcinoma (HCC) is the most common primary malignancy of liver. Distant metastasis to various organs is well known. Skeletal metastasis is also reported to various locations. Vertebral metastasis has been reported mostly to thoracic spine. However, cervical spinal cord involvement leading to cord compression has been reported very rarely in literature. We present a case of 58-year-old male with liver cirrhosis presenting as neck pain. Further work-up revealed metastatic HCC to cervical spinal cord resulting in acute cord compression. Patient has been treated with neurosurgical intervention.

\section{Introduction}

Hepatocellular carcinoma (HCC) is one of the aggressive neoplasms with metastatic potential [1]. Common areas of metastasis include the lung, lymph nodes, bone, and adrenal glands. Although bone metastasis of HCC has been well documented in literature with common locations being ribs, femur, pelvis, and humerus [2], metastasis of HCC to the cervical spinal cord with symptoms of spinal cord compression as the first presentation is very unusual [3-6]. We report a rare clinical scenario of a patient presenting to the hospital with neck pain ultimately leading to the diagnosis of metastatic HCC with cervical spinal cord compression.

\section{Case}

A 58-year-old male presented to the emergency room (ER) of our institution with sharp neck pain of three weeks' duration. The pain was acute in onset, radiating to right shoulder and right lateral aspect of the head. He reported that the pain was associated with numbness in right hand, blurring of vision in the right eye, tinnitus, and dizziness. Patient denied recent trauma to the head and neck. There was no history of weight loss or decreased appetite or change in bladder or bowel habits. Patient also denied headache, vomiting, chest pain, or shortness of breath.

His medical history is significant for cirrhosis of liver secondary to chronic hepatitis $\mathrm{C}$ infection and alcohol. His other medical conditions include essential hypertension and chronic obstructive pulmonary disease. Patient denies undergoing any prior surgical procedures. His mother was diagnosed with breast cancer. He reported smoking one pack of cigarettes for the past 40 years. He has been dependent on alcohol but denied recreational drug use.

On initial evaluation, he was afebrile and hemodynamically stable. Physical examination revealed a tender point in the posterior aspect of neck without any visible anatomical abnormalities. On neurological examination, his mental status was intact and cranial nerve examination was normal. He had increased deep tendon reflexes on right side compared 


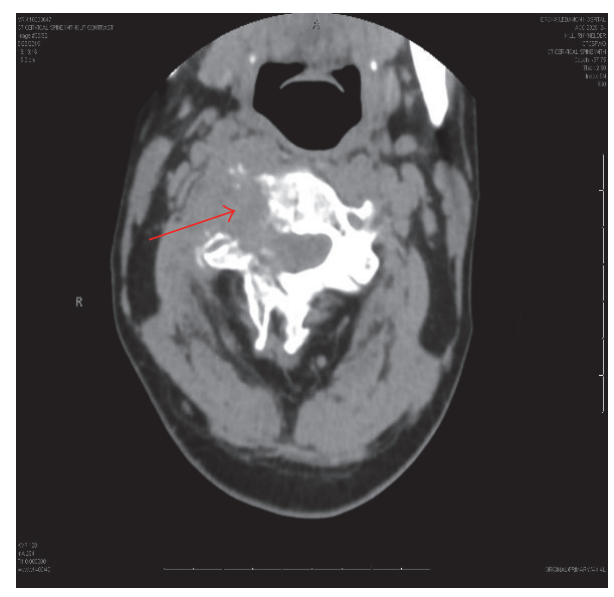

FIGURE 1: CT spine showing a mass at level of C3 with marked osseous destruction.

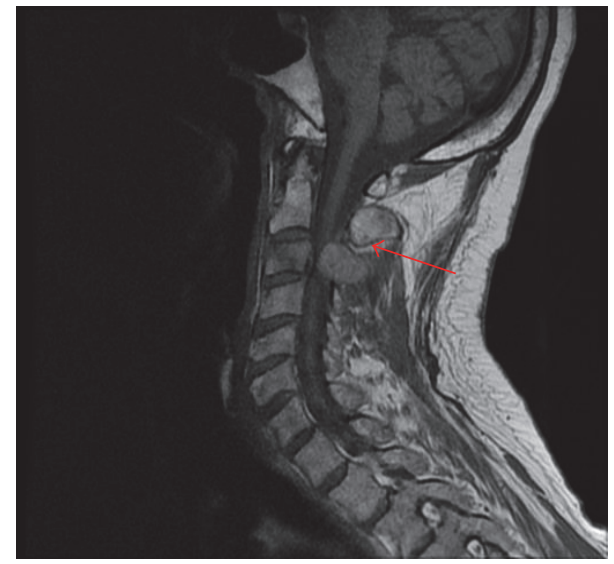

FIGURE 2: MRI of cervical spine: destructive enhancing mass at level of C2-C3 with evidence of spinal cord compression.

to left. There was decreased sensation to touch on the right side and Romberg sign was positive. There were no signs of meningeal irritation. Cardiopulmonary and abdominal examinations were within normal limits.

His laboratory results revealed normal blood counts including platelets. His electrolytes and renal and liver function tests were within normal limits. His hepatitis $\mathrm{C}$ antibody was positive. He was immune to hepatitis A but not immune to hepatitis B.

Computerized tomography (CT) of the cervical spine (Figure 1) revealed a mass at level of C3 with marked osseous destruction and encasement of right vertebral artery. Subsequent magnetic resonance imaging (MRI) of cervical spine (Figure 2) revealed destructive enhancing mass at level of C2C3 with evidence of spinal cord compression. CT and MRI of the brain revealed no acute intracranial hemorrhage or evidence of intracranial neoplasm. CT imaging of abdomen (Figure 3) was significant for a right hemiliver mass. His laboratory results showed elevated alfa-fetoprotein (AFP) of $1167 \mathrm{ng} / \mathrm{mL}$.

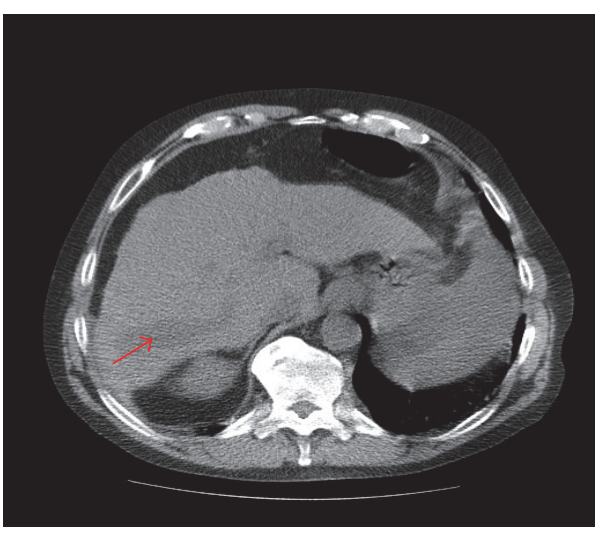

FIGURE 3: CT abdomen showing right hepatic lobe mass.

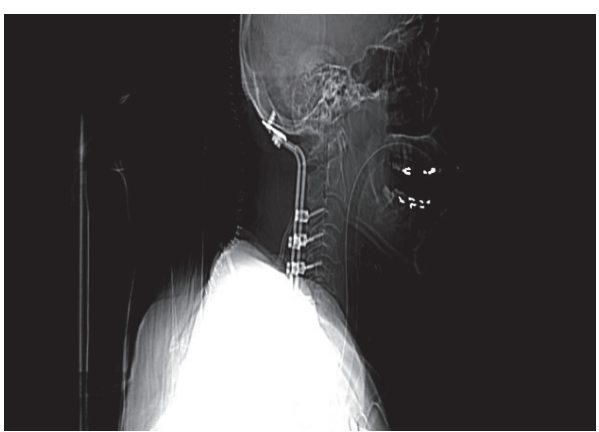

FIGURE 4: CT cervical spine after surgery showing removal of the tumor.

He developed spontaneous intraperitoneal bleeding from tumor leading to drop in hematocrit. He was transferred to critical care unit for close monitoring. He was evaluated by interventional radiologist and underwent imaging guided selective arterial embolization. Subsequently upon stabilization he underwent occipital cervical stabilization and fusion (Figure 4) with excisional biopsy. Histology of the cervical mass confirmed the diagnosis of metastatic hepatocellular carcinoma (Figures 5 and 6). His hospital course was complicated by the development of pneumonia leading to septic shock and death.

\section{Discussion}

HCC is the most common primary tumor of the liver with metastatic potential [1]. Cord compression secondary to metastatic malignancy is seen with breast and lung malignancy [1]. Bone involvement in hepatocellular carcinoma is seen in about 3-20\% of cases in autopsy studies [7]. Most common organs involved in HCC metastasis in the order of decreasing frequency are lung, adrenal gland and bone [8]. While bone metastasis is reported to occur in cases of hepatocellular carcinoma [2], we have noted that presentation as cervical spinal cord compression is extremely unusual.

Review of literature has revealed that so far there have been only few cases of cervical spinal cord compression due to HCC that has been reported (Table 1$)[3-6,9]$. The most 


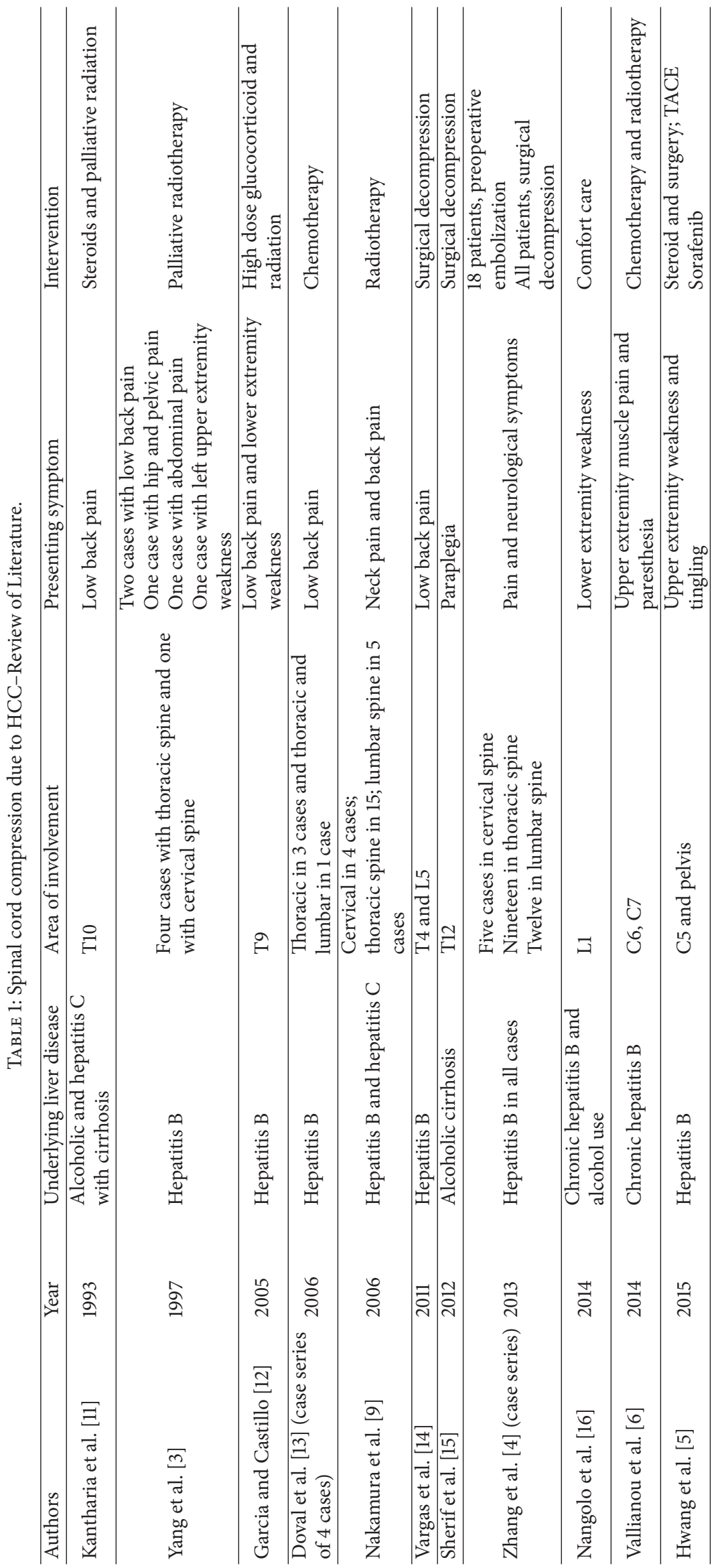




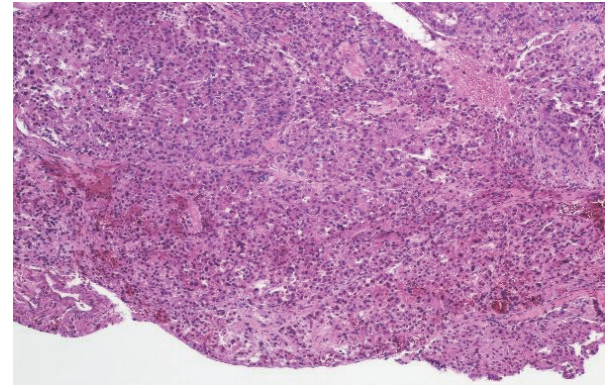

FIGURE 5: Low magnification field of the tumor showing hepatocytes with trabecular pattern of varying thickness separated by sinusoids, features consistent with HCC.

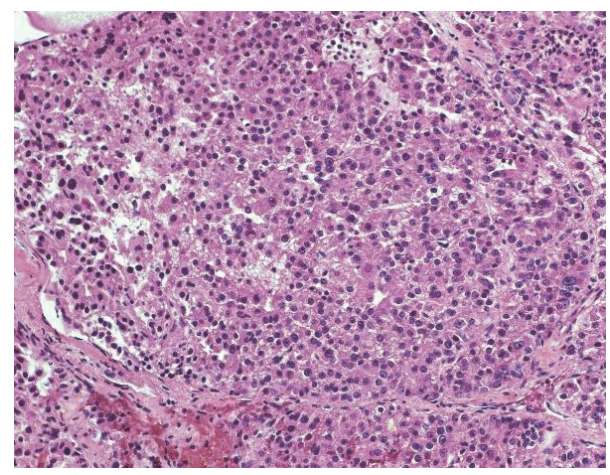

FIGURE 6: High magnification field shows thick trabecula compressed into compact mass surrounded by sinusoids.

common presenting symptom was pain and neurological symptoms. Most of the reported cases in literature received surgical decompression while Sorafenib treatment was also discussed in literature [5].

Cord compression is an acute neurosurgical emergency and metastasis occurring in this setting will probably need surgical intervention. Further treatment of the underlying cancer itself will be based on the patient's overall general condition and severity of liver disease. Cord compression occurs due to invasion of epidural space, most often as a direct extension of vertebral body metastases. There are various routes of the epidural invasion by tumor cells, hematogenous being the most common mode of spread. Hematogenous spread occurs directly or via the involvement of Batson's venous plexuses [10].

Thoracic spinal cord appears to be the most common area of involvement. There are very few cases of HCC in literature reporting spread to the cervical spinal cord and presenting as cord compression. For most of the cases of cervical spinal cord involvement the underlying etiological hepatic risk factor appears to be hepatitis B infection. Most of the cases are reported from the east from areas of increased hepatitis B prevalence [3-5]. Nakamura et al. [9] reported four cases of cervical cord compression due to HCC in whom he evaluated the response of metastasis to radiotherapy. We are also aware in this regard that hepatitis B infection directly has the propensity to lead to hepatocellular malignancy without cirrhosis. This could probably explain the fact that there have been more numbers of reported cases associated with hepatitis B infection. Our patient is a very rare case of cervical spinal cord compression due to HCC secondary to chronic hepatitis $\mathrm{C}$ infection, compounded with alcohol intake.

Improvement in diagnoses and screening of HCC has led to longer overall survival of patients diagnosed with HCC. Consequently, longer survival sheds light on more extrahepatic metastases of HCC and a rise in overall metastatic HCC. Physicians should be aware of the differentials of metastatic malignancy in uncommon sites and uncommon presentations while encountering cases with underlying liver disease. Thus the awareness that cervical spinal cord involvement can occur due to HCC is of significance in initiation of early treatment. This can improve the quality of life and survival of the patients if diagnosed earlier.

\section{Competing Interests}

The authors of the paper declare that there is no conflict of interests regarding the publication of this paper.

\section{References}

[1] S. Katyal, J. H. Oliver III, M. S. Peterson, J. V. Ferris, B. S. Carr, and R. L. Baron, "Extrahepatic metastases of hepatocellular carcinoma," Radiology, vol. 216, no. 3, pp. 698-703, 2000.

[2] J. E. Kuhlman, E. K. Fishman, P. K. Leichner, D. Magid, S. E. Order, and S. S. Siegelman, "Skeletal metastases from hepatoma: frequency, distribution, and radiographic features," Radiology, vol. 160, no. 1, pp. 175-178, 1986.

[3] W. T. Yang, W. Yeo, S. F. Leung, Y. L. Chan, P. J. Johnson, and C. Metreweli, "MRI and CT of metastatic hepatocellular carcinoma causing spinal cord compression," Clinical Radiology, vol. 52, no. 10, pp. 755-760, 1997.

[4] D. Zhang, W. Xu, T. Liu et al., "Surgery and prognostic factors of patients with epidural spinal cord compression caused by hepatocellular carcinoma metastases: retrospective study of 36 patients in a single center," Spine, vol. 38, no. 17, pp. E1090E1095, 2013.

[5] S. W. O. Hwang, J. E. U. Lee, J. M. I. Lee et al., "Hepatocellular carcinoma with cervical spine and pelvic bone metastases presenting as unknown primary neoplasm," The Korean Journal of Gastroenterology, vol. 66, no. 1, pp. 50-54, 2015.

[6] N. G. Vallianou, P. Gounari, A. Skourtis, and C. Vourlakou, "Cervical mass as the presenting manifestation of hepatocellular carcinoma," Hippokratia, vol. 18, no. 3, pp. 285-287, 2014.

[7] H. A. Edmondson and P. E. Steiner, "Primary carcinoma of the liver. A study of 100 cases among 48,900 necropsies," Cancer, vol. 7, no. 3, pp. 462-503, 1954.

[8] T. Nakashima, K. Okuda, M. Kojiro et al., "Pathology of hepatocellular carcinoma in Japan. 232 consecutive cases autopsied in ten years," Cancer, vol. 51, no. 5, pp. 863-877, 1983.

[9] N. Nakamura, H. Igaki, H. Yamashita et al., "A retrospective study of radiotherapy for spinal bone metastases from hepatocellular carcinoma (HCC)," Japanese Journal of Clinical Oncology, vol. 37, no. 1, pp. 38-43, 2006. 
[10] O. V. Batson, "The function of the vertebral veins and their role in the spread of metastases," Annals of Surgery, vol. 112, no. 1, pp. 138-149, 1940.

[11] B. Kantharia, R. Nizam, H. Friedman, and S. Vardan, "Case report: spinal cord compression due to metastatic hepatocellular carcinoma," American Journal of the Medical Sciences, vol. 306, no. 4, pp. 233-235, 1993.

[12] V. A. Garcia and R. Castillo, "Asymptomatic advanced hepatocellular carcinoma presenting with spinal cord compression," Digestive Diseases and Sciences, vol. 50, no. 2, pp. 308-311, 2005.

[13] D. C. Doval, K. Bhatia, A. K. Vaid et al., "Spinal cord compression secondary to bone metastases from hepatocellular carcinoma," World Journal of Gastroenterology, vol. 12, no. 32, pp. 5247-5252, 2006.

[14] J. Vargas, M. Gowans, W. A. Vandergrift, J. Hope, and P. Giglio, "Metastatic hepatocellular carcinoma with associated spinal cord compression," The American Journal of the Medical Sciences, vol. 341, no. 2, pp. 148-152, 2011.

[15] K. A. Sherif, W. Frandah, S. Graham, and M. Phy, "Hepatocellular carcinoma as unusual cause for paraplegia," Journal of Clinical Oncology, vol. 30, no. 31, pp. e303-e305, 2012.

[16] H. T. Nangolo, L. Roberto, I. L. Segamwenge, A. Voigt, and F. Kidaaga, "Spinal cord compression: an unusual presentation of hepatocellular carcinoma," Pan African Medical Journal, vol. 19, article 363, 2014. 


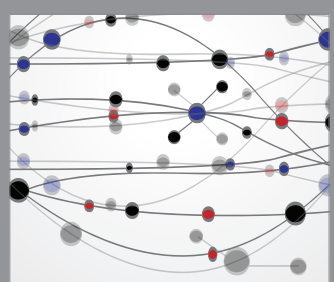

The Scientific World Journal
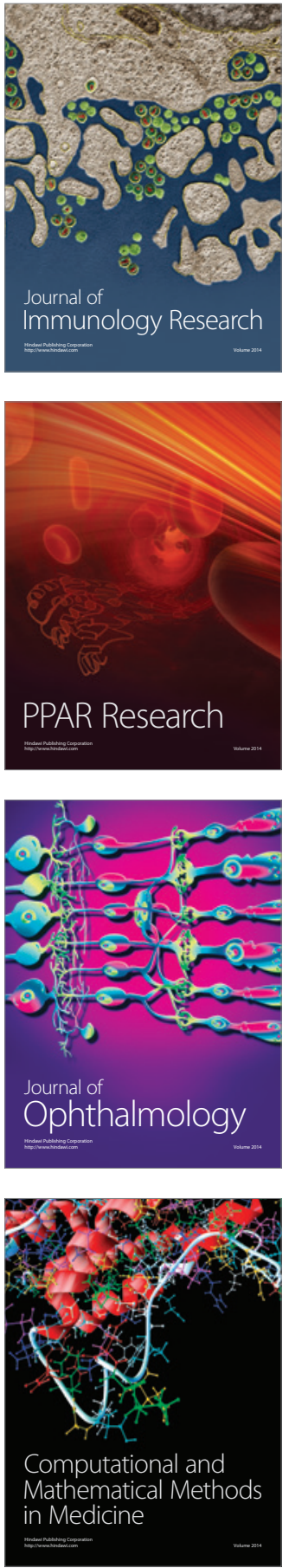

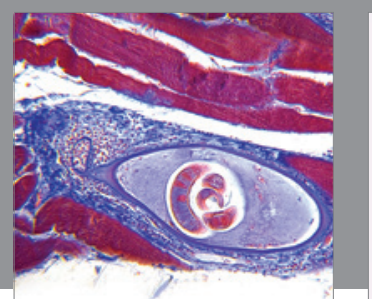

Gastroenterology Research and Practice
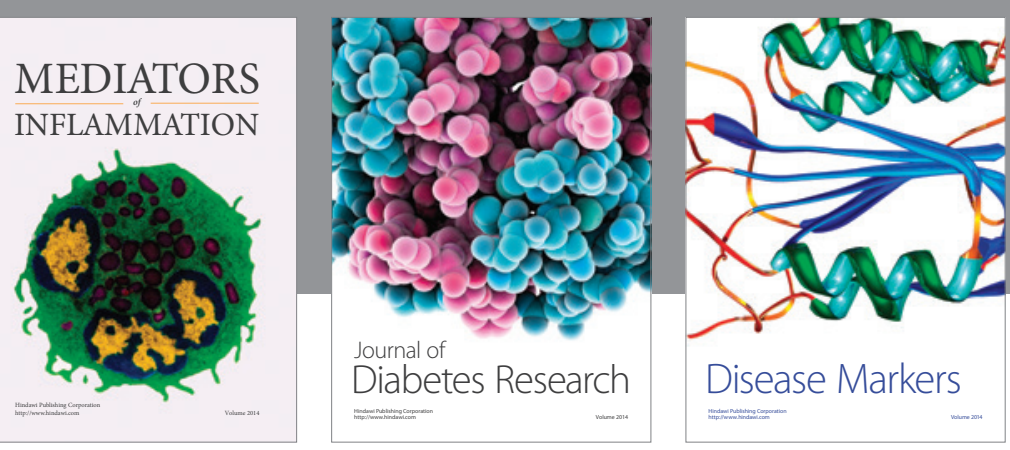

Disease Markers

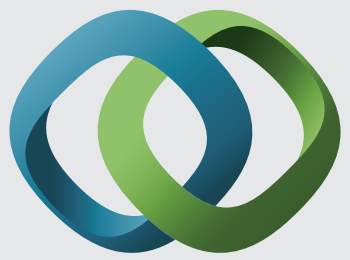

\section{Hindawi}

Submit your manuscripts at

https://www.hindawi.com
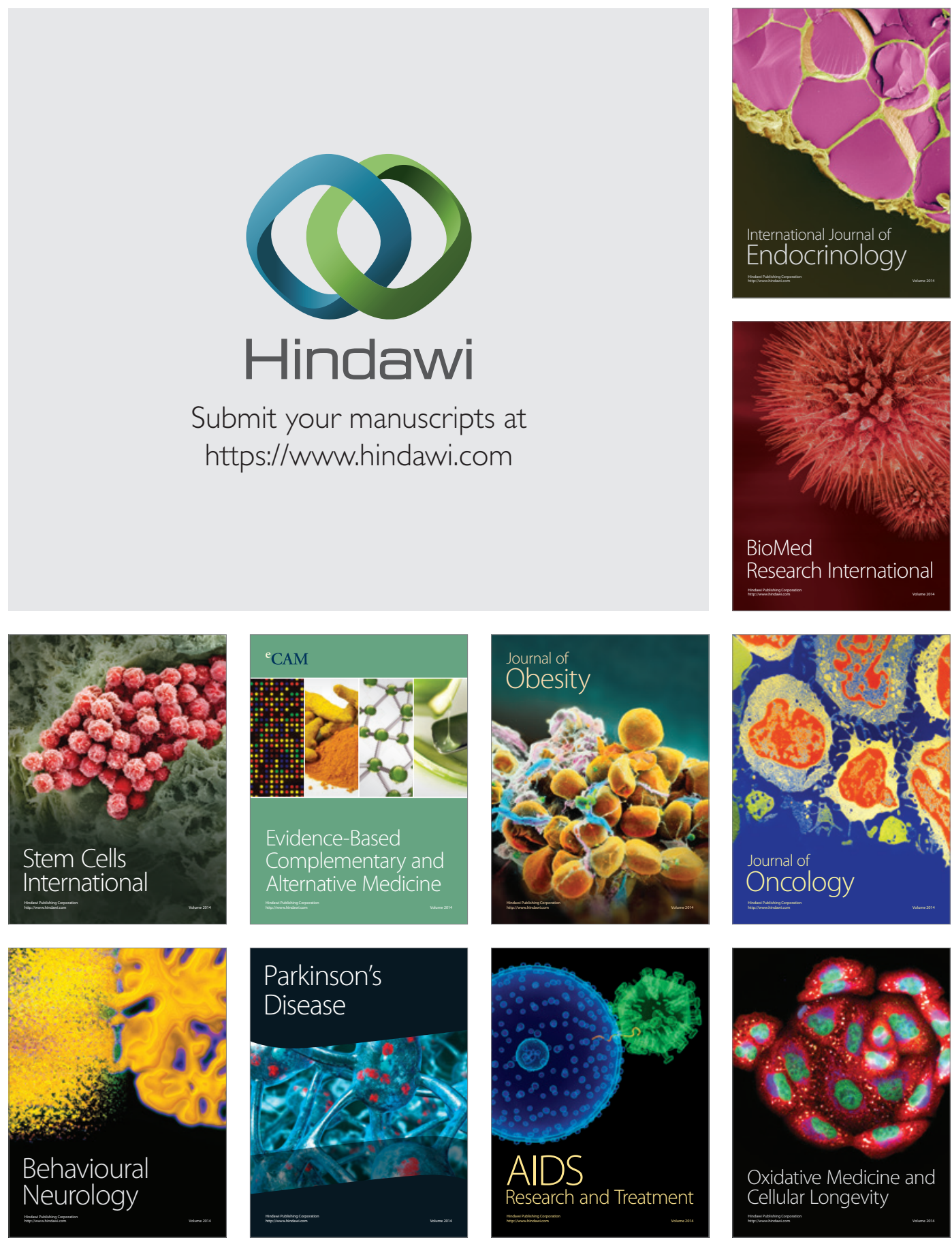